PROCEEDINGS OF THE

AMERICAN MATHEMATICAL SOCIETY

Volume 129, Number 12, Pages 3539-3541

S 0002-9939(01)06252-9

Article electronically published on July 2, 2001

\title{
THE PRODUCT OF A GÂTEAUX DIFFERENTIABILITY SPACE AND A SEPARABLE SPACE IS A GÂTEAUX DIFFERENTIABILITY SPACE
}

\author{
LIXIN CHENG AND MARIÁN FABIAN
}

(Communicated by Jonathan M. Borwein)

\begin{abstract}
This paper shows that the product of a Gâteaux differentiability space and a separable Banach space is again a Gâteaux differentiability space.
\end{abstract}

A Banach space is called a Gâteaux differentiability space if every convex continuous function on it is Gâteaux differentiable at the points of a dense set. For more information about this class and its relatives - weak Asplund and Asplund spaces - see [LP, [NP, $[\mathrm{A},[\mathrm{P},[\mathrm{F}],[\mathrm{DGZ}, \mathrm{G}]$. Relatively little is known about the stability of the class of the Gâteaux differentiability spaces. In the late eighties, the second named author showed that if $X$ is a Gâteaux differentiability space, then so is the product of $X$ and the real line; see [P, Proposition 6.5]. The aim of this note is to extend this fact to separable spaces.

Theorem. Let $X$ be a Gâteaux differentiability space and let $Y$ be an arbitrary separable Banach space. Then $X \times Y$ is also a Gâteaux differentiability space.

Proof. We shall elaborate Ioffe's penalty method used in the proof of $[\mathrm{P}$, Proposition $6.5]$, while incorporating some ideas from Klee $[\mathbf{K}]$.

Let $B_{X}$ and $B_{Y}$ denote the closed unit balls in $X$ and $Y$ respectively. Let $f: X \times Y \rightarrow \mathbb{R}$ be a convex continuous function. Let $\Omega$ be any nonempty open set in $X \times Y$. We have to find a point in $\Omega$ where $f$ is Gâteaux differentiable. By a suitable shift of variables we can arrange things so that $(0,0) \in \Omega$. Since $f$ is continuous at $(0,0)$, we can find $\Delta>0$ so that $\Omega$ contains $S=2 \Delta B_{X} \times 2 \Delta B_{Y}$ and that $f$ is bounded on this "square". Let $M>0$ be a constant such that $|f(x, y)|<M$ whenever $(x, y) \in 2 \Delta B_{X} \times 2 \Delta B_{Y}$. Let $\left\{y_{i} ; i \in \mathbb{N}\right\}$ be a dense subset in $\Delta B_{Y}$. Define $g: 2 \Delta B_{X} \rightarrow \mathbb{R}$ as

$g(x)=\sup \left\{f\left(x, \sum 2^{-i} \alpha_{i} y_{i}\right)-\tan \left(\sum \alpha_{i}^{2}\right) ; \alpha_{i} \in \mathbb{R}, \sum \alpha_{i}^{2}<\frac{\pi}{2}\right\}, \quad x \in 2 \Delta B_{X}$.

Received by the editors March 16, 2000.

2000 Mathematics Subject Classification. Primary 46B20, 46G05, 26E15, 58C20.

Key words and phrases. Convex function, Banach space, Gâteaux differentiability space.

The first author was supported by NSFC 10071063, F00021.

The second author was supported by GA ČR 201-98-1449, GA ČR 201/01/1198, and AV 1019003. 
Here and below the symbol ' $\sum$ ' will stand for summation for $i$ from one to infinity. Note that for every $x \in 2 \Delta B_{X}$ we have

$$
-M<f(x, 0) \leq g(x) \leq \sup \left\{f\left(x, \sum 2^{-i} \alpha_{i} y_{i}\right) ; \alpha_{i} \in \mathbb{R}, \sum \alpha_{i}^{2}<\frac{\pi}{2}\right\} \leq M .
$$

Hence $g$ is a convex bounded function on $2 \Delta B_{X}$. It follows by [P, Proposition 1.6] that $g$ is Lipschitz on $\Delta B_{X}$. Then it is well known that $g$, restricted to $\Delta B_{X}$, has an extension to a convex Lipschitz function defined on all of $X$, which we also denote by $g$.

Since $X$ is a Gâteaux differentiability space, there is $x_{0} \in X$, with $\left\|x_{0}\right\|<\Delta$, where the function $g$ is Gâteaux differentiable. Let $\alpha_{i, n} \in \mathbb{R}, i, n \in \mathbb{N}$, be such that $\sum_{i=1}^{\infty} \alpha_{i, n}^{2}<\frac{\pi}{2}$ for every $n \in \mathbb{N}$ and

$$
f\left(x_{0}, \sum 2^{-i} \alpha_{i, n} y_{i}\right)-\tan \left(\sum \alpha_{i, n}^{2}\right) \rightarrow g\left(x_{0}\right) \quad \text { as } n \rightarrow \infty .
$$

As $f$ is bounded on $S$ we derive that

$$
\liminf _{n \rightarrow \infty} \tan \left(\sum \alpha_{i, n}^{2}\right)<+\infty \text { and } \liminf _{n \rightarrow \infty} \sum \alpha_{i, n}^{2}<\frac{\pi}{2} .
$$

A diagonal process allows us to assume that $\alpha_{i, n} \rightarrow \alpha_{i}$ as $n \rightarrow \infty$ for every $i \in \mathbb{N}$. Then

$$
\sum \alpha_{i}^{2}=\lim _{N \rightarrow \infty} \sum_{i=1}^{N} \alpha_{i}^{2}=\lim _{N \rightarrow \infty} \lim _{n \rightarrow \infty} \sum_{i=1}^{N} \alpha_{i, n}^{2} \leq \liminf _{n \rightarrow \infty} \sum_{i=1}^{\infty} \alpha_{i, n}^{2}<\frac{\pi}{2} .
$$

Setting $y_{0}=\sum 2^{-i} \alpha_{i} y_{i}$, we observe that $y_{0} \in \Delta B_{Y}$. Now, for any $N \in \mathbb{N}$ we have

$$
\begin{aligned}
\limsup _{n \rightarrow \infty} & \left\|\sum_{i=1}^{\infty} 2^{-i} \alpha_{i, n} y_{i}-y_{0}\right\| \leq \limsup _{n \rightarrow \infty}\left\|\sum_{i=1}^{N} 2^{-i}\left(\alpha_{i, n}-\alpha_{i}\right) y_{i}\right\| \\
& +\limsup _{n \rightarrow \infty}\left\|\sum_{i=N+1}^{\infty} 2^{-i}\left(\alpha_{i, n}-\alpha_{i}\right) y_{i}\right\| \\
\leq & \limsup _{n \rightarrow \infty}\left(\sum_{i=N+1}^{\infty} 2^{-2 i}\right)^{1 / 2}\left(\sum_{i=N+1}^{\infty}\left(\alpha_{i, n}-\alpha_{i}\right)^{2}\right)^{1 / 2} \\
\leq & \frac{1}{\sqrt{3}} 2^{-N} \limsup _{n \rightarrow \infty}\left(2 \sum_{i=N+1}^{\infty} \alpha_{i, n}^{2}+2 \sum_{i=N+1}^{\infty} \alpha_{i}^{2}\right)^{1 / 2}<\sqrt{\frac{2 \pi}{3}} 2^{-N},
\end{aligned}
$$

and hence $\left\|\sum_{i=1}^{\infty} 2^{-i} \alpha_{i, n} y_{i}-y_{0}\right\| \rightarrow 0$ as $n \rightarrow \infty$. Thus, using the continuity of $f$ we have

$$
\begin{aligned}
g\left(x_{0}\right) & =\lim _{n \rightarrow \infty}\left[f\left(x_{0}, \sum 2^{-i} \alpha_{i, n} y_{i}\right)-\tan \left(\sum \alpha_{i, n}^{2}\right)\right] \\
& =f\left(x_{0}, y_{0}\right)-\tan \left(\liminf _{n \rightarrow \infty} \sum \alpha_{i, n}^{2}\right) \\
& \leq f\left(x_{0}, y_{0}\right)-\tan \left(\sum \alpha_{i}^{2}\right) \leq g\left(x_{0}\right) .
\end{aligned}
$$

Now, fix arbitrary $h \in X$ and $j \in \mathbb{N}$. Then for $t>0$ small enough we have

$$
\sum_{i \neq j} \alpha_{i}^{2}+\left(\alpha_{j} \pm 2^{j} t\right)^{2}<\frac{\pi}{2}
$$


and hence

$$
\begin{aligned}
0 \leq & f\left(x_{0}+t h, y_{0}+t y_{j}\right)+f\left(x_{0}-t h, y_{0}-t y_{j}\right)-2 f\left(x_{0}, y_{0}\right) \\
\leq & g\left(x_{0}+t h\right)+\tan \left(\sum_{i \neq j} \alpha_{i}^{2}+\left(\alpha_{j}+2^{j} t\right)^{2}\right) \\
& +g\left(x_{0}-t h\right)+\tan \left(\sum_{i \neq j} \alpha_{i}^{2}+\left(\alpha_{j}-2^{j} t\right)^{2}\right) \\
& -2 g\left(x_{0}\right)-2 \tan \left(\sum \alpha_{i}^{2}\right)=o(t)+o(t)
\end{aligned}
$$

since $g$ is Gâteaux differentiable at $x_{0}$. (This is true also if $\sum \alpha_{i}^{2}=0$ since $\tan \tau<$ $2 \tau$ for small $\tau>0$.)

Finally, recalling that $\left\{y_{i} ; i \in \mathbb{N}\right\}$ is a dense set in $\Delta B_{Y}$, and using the local Lipschitzness of $f[\mathrm{P}$, Proposition 1.6], we have that

$$
f\left(x_{0}+t h, y_{0}+t k\right)+f\left(x_{0}-t h, y_{0}-t k\right)-2 f\left(x_{0}, y_{0}\right)=o(t)
$$

for every $h \in X$ and every $k \in Y$. Since $f$ is convex, this is equivalent to the Gâteaux differentiability of $f$ at $\left(x_{0}, y_{0}\right)$.

\section{ACKNOWLEDGEMENT}

We thank J. M. Borwein and V. Zizler for helpful discussions.

\section{REFERENCES}

[A] E. Asplund, Fréchet differentiability of convex functions, Acta Math. 121 (1968), 31-47. MR 37:6754

[DGZ] R. Deville, G. Godefroy, and V. E. Zizler, Smoothness and renormings in Banach spaces, Pitman Monographs, No. 64, 1993. MR 94d:46012

[F] M. J. Fabian, Gâteaux differentiability of convex functions and topology. Weak Asplund spaces, J. Wiley \& Sons, Interscience, New York, 1997. MR 98h:46009

[G] J. R. Giles, Convex analysis with application in differentiation of convex functions, Research Notes in Math., No. 58, Pitman, Boston-London-Melbourne, 1982. MR 83g:46001

$[\mathrm{K}] \quad \mathrm{V}$. Klee, Some new results on smoothness and rotundity in normed linear spaces, Math. Annalen 139 (1959), 51-63. MR 22:5879

[LP] D. G. Larman and R. R. Phelps, Gâteaux differentiability of convex functions on Banach spaces, J. London Math. Soc. 20 (1979), 115-127. MR 80m:46017

[NP] I. Namioka and R. R. Phelps, Banach spaces which are Asplund spaces, Duke Math. J. 42 (1975), 735-750. MR 52:11544

[P] R. R. Phelps, Convex functions, monotone operators, and differentiability, Lecture Notes in Math., No. 1364, Springer Verlag, 1989; 2nd Edition, 1993. MR 90g:46063 MR 94f: 46055

Department of Mathematics, Xiamen University, Xiamen 361005, People's Republic OF CHINA

Mathematical Institute, Czech Academy of Sciences, Žitná 25, 11567 Prague 1, Czech REPUBLiC 